\title{
The navigator of new breaker-vibro ripper SV-80
}

\author{
Hu Kaijun ${ }^{1}$, Yang Guoping ${ }^{1}$, Cao Guojun ${ }^{2}$, Wang Kai ${ }^{1}$, Wang Kaile ${ }^{1}$ \\ ${ }^{1}$ Automotive Engineering College, Shanghai University of Engineering Science, Shanghai, China \\ ${ }^{2}$ Shanghai Shining Mechanical Technology Co, Ltd. Shanghai, China
}

\section{Email address:}

dfhukaijun@163.com (Hu Kaijun),ygpljyl@163.com (Yang Guoping),875977150@qq.com (Cao Guojun), 1158698182@qq.com (Wang Kai),762349464@qq.com (Wang Kaile)

\section{To cite this article:}

Hu Kaijun, Yang Guoping, Cao Guojun, Wang Kai, Wang Kaile. The Navigator of New Breaker-Vibro Ripper SV-80. Science Journal of Energy Engineering. Vol. 2, No. 4, 2014, pp. 32-35. doi: 10.11648/j.sjee.20140204.11

\begin{abstract}
Make a introduce of vibro ripper SV-80 focus on technical parameters, structural features and innovations. It is the largest vibro ripper in the world and with rational construction as well as advanced performance, SV- 80 includes low noise, strong force, environmental protection, high efficiency, flexible mobility and other nice characters. It's a new type of breaker and must bring about a revolution in crushing machinery.
\end{abstract}

Keywords: Vibrate, Vibro Ripper, SV-80, Efficiency

\section{Introduction}

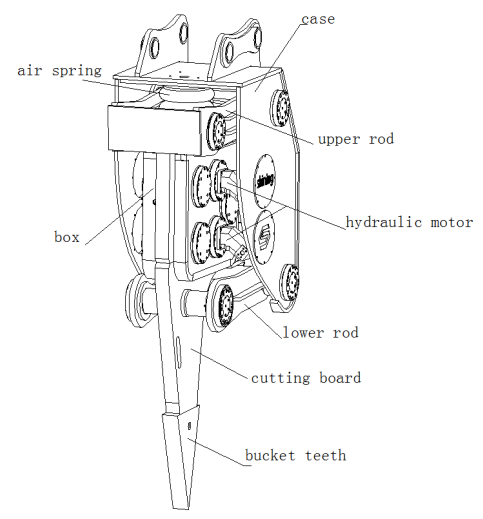

Fig. 1. Vibro ripper $S V-80$

As is shown in Fig. 1, vibro ripper SV-80 belongs to crushing appliance in engineering machinery[1], and it's the largest vibro ripper in the world now. Compared with piston breaker[2], SV-80 can turn hydraulic energy into mechanical energy instantly. It using two hydraulic motors to turn two pairs of eccentric blocks separately and synchronously, which would make a centrifugal force and called vibration, this vibration passed to rock by cutting board and crush them instantly. SV-80 can complete complicated tasks in various kinds of environment because of its powerful feature. As a result of vibro ripper $\mathrm{SV}-80$ has a prominence of energy saving, environmental protection and high efficiency, it would be applicable for some large crushing engineering like large rocks broken, mine excavation and building construction, especially in the field of municipal construction and concrete broken[3]. According to the market research information, SV-80 is not only the largest vibro ripper but also a rare equipment in crushing machinery, its prominent performance will help it to exploit a huge market in the world[4].

\section{Main Technical Parameters}

SV-800 has the characters of security, reliability and high efficiency[5,6], it mainly include case, rod(upper rod and lower rod), air spring and vibrator. Vibrator is the heart of SV-80 and it includes box, hydraulic motor, vibration unite, cutting board and bucket teeth. The following table. 1 reveals the main parameters of vibro ripper SV-80: 
Table 1. The main Parameters of vibro ripper SV-80

\begin{tabular}{|c|c|c|c|c|c|}
\hline Total performance & & Hydraulic motor (HYDRO L & EUC)M108 & Air spring (Shanghai wangfu)DN320 & \\
\hline Excavator tons $(\mathrm{T})$ & $70-100$ & Displacement(cc/rev) & 108.3 & \multirow{5}{*}{ Medium } & \multirow{5}{*}{$\mathrm{N}_{2}$} \\
\hline Max vibration $(\mathrm{KN})$ & 820 & Continuous max. speed(rpm) & 4000 & & \\
\hline Frequency(Hz) & $25-30$ & Intermittent max. speed(rpm) & 4400 & & \\
\hline Total weight(kg) & 8500 & Max. Flow absorbed(1/min) & 435 & & \\
\hline Noise $(\mathrm{db})$ & $58-76$ & Min./Max. temperature $\left({ }^{0} \mathrm{c}\right)$ & $-25 / 110$ & & \\
\hline Working speed(r/min) & $1500-1800$ & Torque(N.m/bar) & 1.72 & \multirow{4}{*}{ Working pressure } & \multirow{4}{*}{$\begin{array}{l}0.7 \\
\mathrm{Mpa}\end{array}$} \\
\hline Working flow(1/min) & $290-360$ & Max pressure continuous(bar) & 400 & & \\
\hline Working pressure(bar) & $250-280$ & Max pressure peak(bar) & 450 & & \\
\hline Return pressure(bar) & 6 & Weight(kg) & 23 & & \\
\hline
\end{tabular}

\section{Structure and Characteristics}

\subsection{Case of $S V-80$}

The weight of case is about $2062 \mathrm{~kg}$, it is welded by high-strength low alloy steel Q460, the mechanical properties and material composition of Q460 are in table. 2, it is suitable for SV-80 case because of its high strength.

To maximize protect box and hydraulic motor, SV-80 case use a half-closed space, meanwhile, it sustains the force from vibrator. The top of the case connect with excavator[7], the interior connect with rod by axle, and axle is locate in the side of the case by joint bearing. On account of SV-80 makes a violent vibration in the work, the rod not only endure a high exciting force but also make a swing frequently, which make joint bearing endures high axial force and radial force, but joint bearing can make a self-adjustment moving to some extent and it can lubricate by itself because of the composite on its inside circle, which has made vibro ripper SV-80 working in some extreme conditions as usual and it has improved the capability.

Table 2. Material properties of high-strength low alloy steel

\begin{tabular}{|c|c|c|c|c|c|c|c|c|c|c|}
\hline \multirow{4}{*}{$\begin{array}{l}\text { Grade: } \\
\text { Q460 }\end{array}$} & \multirow{4}{*}{$\begin{array}{l}\text { Mechanical } \\
\text { properties }\end{array}$} & \multicolumn{3}{|c|}{ Yield strength $\sigma / \mathrm{Mpa}$} & \multicolumn{3}{|c|}{ Tensile strength/Mpa } & \multicolumn{3}{|c|}{ Elongation $\delta_{5} / \%$} \\
\hline & & 460 & & & $550-720$ & & & 17 & & \\
\hline & & $\Omega(\mathrm{C})$ & $\omega(\mathrm{Si})$ & $\omega(\mathrm{Mn})$ & $\omega(\mathrm{S})$ & $\omega(\mathrm{P})$ & $\omega(\mathrm{Cr})$ & $\omega(\mathrm{Ni})$ & $\omega(\mathrm{Ti})$ & $\omega(\mathrm{Nb})$ \\
\hline & & $\leq 0.2$ & $\leq 0.55$ & $1.0 \sim 1.7$ & $\leq 0.035$ & $\leq 0.035$ & $\leq 0.7$ & $\leq 0.7$ & $0.02 \sim 0.2$ & $0.015 \sim 0.06$ \\
\hline
\end{tabular}

\subsection{Shock Absorber}

As is shown in Fig. 2, the shock absorber is an air spring DN320 with two-bend design which made in Shanghai WangFu rubber factory. The self-resonant frequency of DN320 is almost constant under any load with nonlinear characteristic that rigidity changes with load, so that the spring mechanism can keep the same. DN320 have good performance of absorbing high frequency vibration and sound insulating. And its carry both axial load and radical load, also can get different load ability by adjusting the inner pressure to meet the needs of various load and so as to achieve the flexible transmission of bearing capacity. Air spring DN320 can also decrease the vibration to excavator and provides a comfortable condition to the driver.

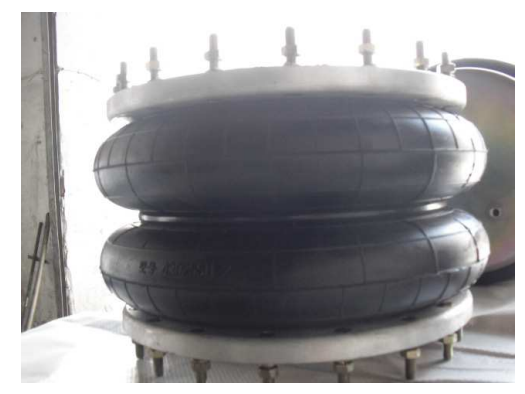

Fig. 2. Air spring DN320

\subsection{Rods}

Rods are essential connections between case and vibrator, they include upper rod and lower rod, they are all made by Q460. The main side of rods connect with vibrator by joint bearing and the other side of rods connect with case by axle, they compose a four bar linkage and vibrator can moving vertically in the case, so it can ensure the working point accurately and promote efficiency in the work.

\subsection{Vibrator}

Vibrator is the core unite of vibro ripper SV-80, and there are two pairs of eccentric blocks meshed separately in it. SV-80 employs superimposed vibration technology and drave by two hydraulic motors[8]. Fig. 3 shows the principle of vibrator, SV-80 can make a superimposed force in vertical direction but the force would be counteracted in horizontal direction. Vibrator vibrates heavily in vertical orientation as well as crush the rock. In order to ensure working performance, box and cutting board are all made by Q460 too[9], bucket teeth using rock teeth and imports from the United States ESCO. Eccentric blocks made in integrated manufacturing and adopt helical gear transmission with low noise, constant stability, high efficiency, longevity etc, which can promote SV-80 performance prominently. 


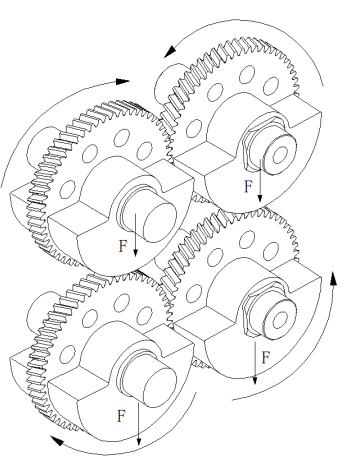

Vertical direction

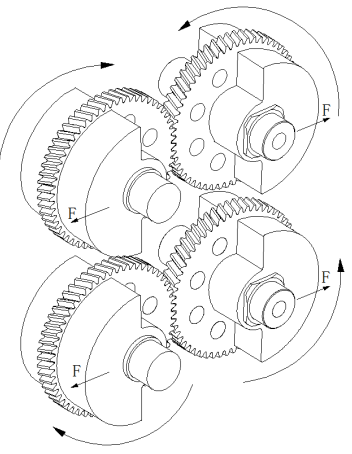

Horizontal direction
Fig. 3. Vibration unite

\subsection{Hydraulic motor}

Hydraulic motor transform hydraulic flow into rotating speed and hydraulic pressure into mechanical torque.Motor rotating speed is proportional to the flow which is supplied to it. Torque produced is proportional to the hydraulic pressure the motor receives.To ensure SV-80 work normally in any condition, we use LEDUC M108 hydraulic motor to drive eccentric blocks.M108 has a high quality materials and workmanship,the design choices highlighted below ensure the remarkable reliability and long service life of motor.Fig.4 shows the advantages of LEDUC M108 in structure.

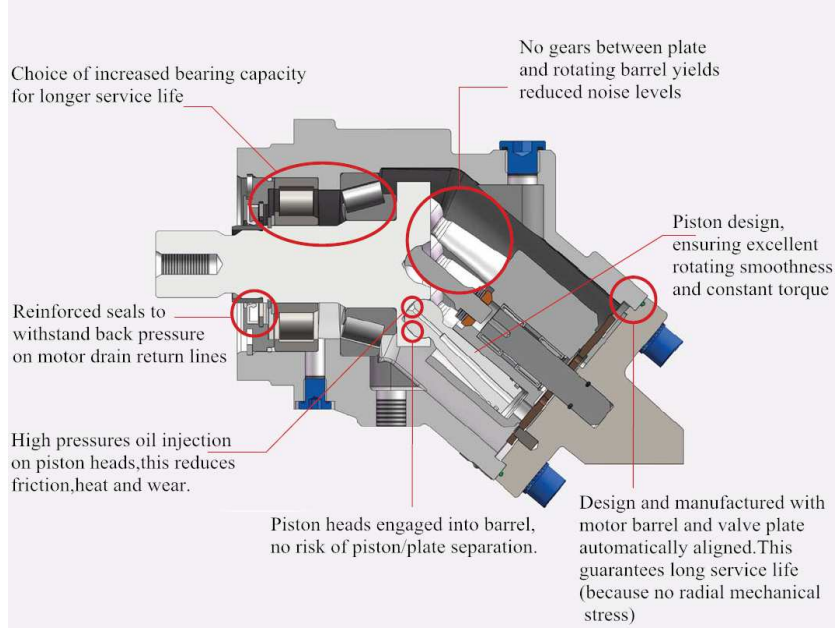

Fig. 4. Advantages of LEDUC M108

Meanwhile,M108 as the power source of SV-80,its efficiency has a significant effect on vibro ripper in dynamic performance. Fig.5 shows the relationship between efficiency and pressure at a speed of $1500 \mathrm{rpm}$. When the pressure is between 250bar and 280 bar,the efficiency is more than $90 \%$, which effectively ensure the mobility of vibro ripper.

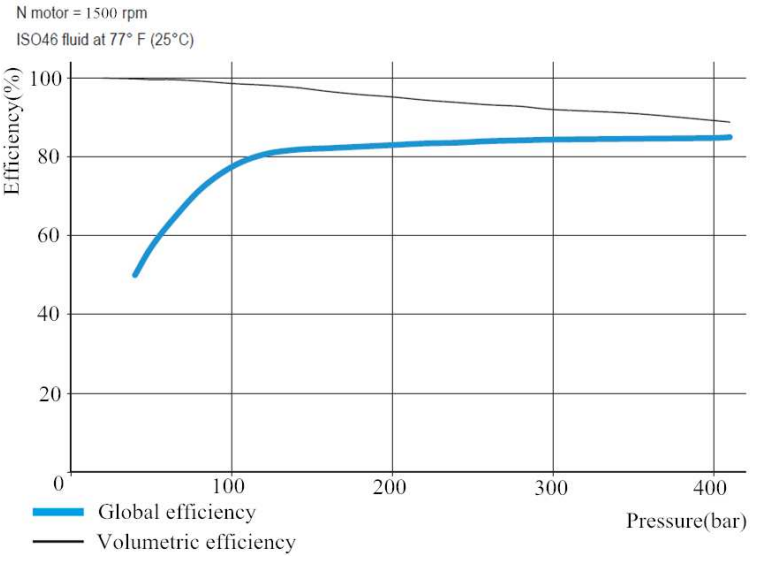

Fig. 5. Efficiency of hydraulic motor M108

\section{The Performance Characteristic of Vibro Ripper SV-80}

As stated above, SV-80 using imports and employs superimposed vibration technology to ensure its quality and reliability, it has made a great improvement to its work. Further more, SV-80 will bring about a revolution in crushing machine because of its excellent performance.

\subsection{The Innovation of Vibro Ripper SV-80}

SV-80 differs from traditional breaker which employs reciprocating theory, SV-80 using four eccentric blocks rotating in the box and produce a high frequency to hit the rock. The weight of eccentric blocks is about $640 \mathrm{~kg}$ and revolution various from $1500 \mathrm{r} / \mathrm{min}$ to $1800 \mathrm{r} / \mathrm{min}$, it produces a high centrifugal force from $600 \mathrm{KN}$ to $800 \mathrm{KN}$. According to Fig. 6 we can see that vibration various with revolution obviously and SV-80 always work accurately to one point, meanwhile, it enlarges the gap and makes a second destruction. Therefore, vibro ripper SV-80 has a high efficiency than traditional breaker and now it has applied a patent in China.

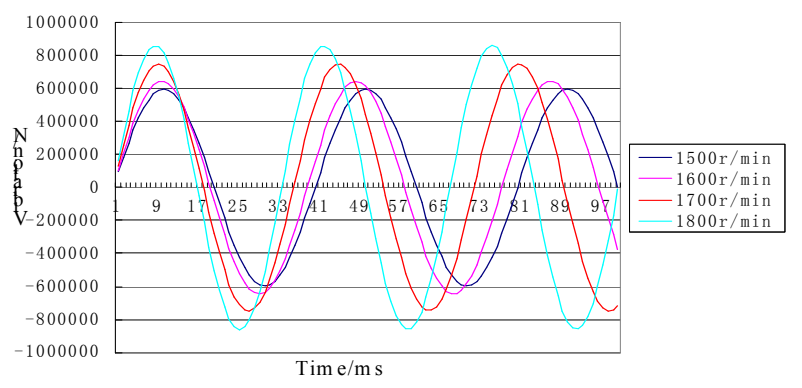

Fig. 6. Vibration in various revolution

\subsection{The Performance Characteristic Analysis}

Vibro ripper SV-80 is aimed at mining initially[10], but on account of its advantages with economical performance[11], maintenance free operation, low noise, environmental protection etc, it becomes wide spread to to the other construction area like urbanization. SV-80 has 
decreased 90 percent of vibration by shock absorb and there is no more damage to excavator. In terms of general maintenance, there is almost no hydraulic oil leak and only need 3-8 accelerator from excavator to work[12]. To maintain the normal performance of SV-80, we just need to replace some vulnerable part termly,like shock absorber and bucket teeth . In the aspect of vibration noise, SV-80 has only about $58-76 \mathrm{db}$ and it meets the urban standard. It's nearly no dust and muck spread abroad. SV-80 can work in 10 meters depth water with no modification because of its totally-enclosed box. There is no dirty oil flow from SV-80 to excavator and prill in vibrator can't flow into excavator hydraulic system, this is an important character in vibro ripper SV-80.

\subsection{Significance to Public Safety}

On the aspect of efficiency, SV-80 can triumph over piston breaker in most working condition and even can completely replace blast according to requirements of operation in some cases. It has a great significance to improve the public security.

Fig.7 and Fig. 8 show the efficiency index that we get from the proving ground preliminarily.vibro ripper compared with piston breaker and blast in rock working condition, which can play an important guiding role for engineers in job site.

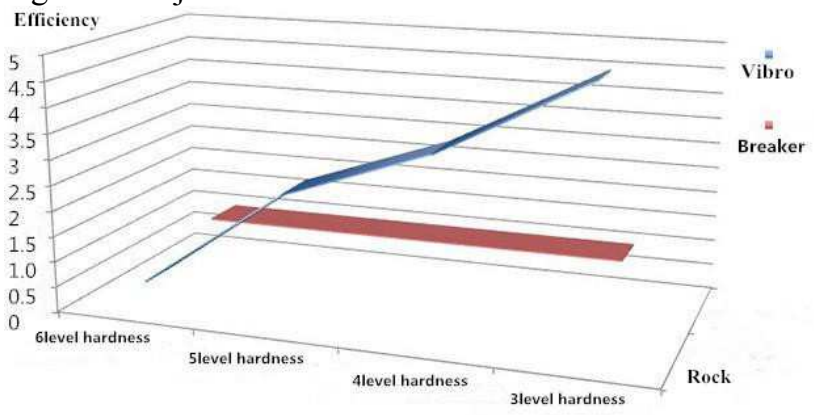

Fig. 7. Vibro compares with Breaker

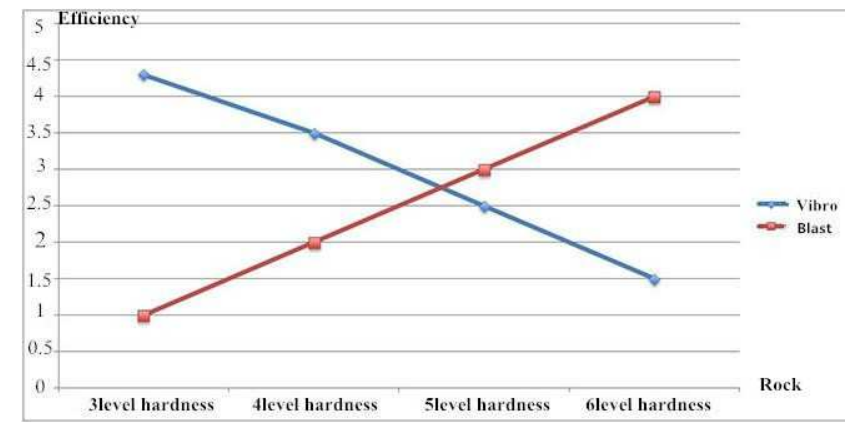

Fig.8. Vibro compares with Blast

\section{Conclusion}

Through market research and analysis.Vibro ripper SV-80 haven't equipped largely in the world,and there are only a few countries developing the similar products currently,like Spanish Tabe,South Korea Dadong,Shanghai
Fenyi and Spanish Xcentric,but their performance are all inferior to SV-80.On account of SV-80 excellent performance in the experimental stage, especially in the field of instead of blast operation and municipal construction, it is doomed to have a strong competitive advantage in the international market and have a large space for development in the future of crushing machinery with its good cost performance.

At present, SV-80 is a new breaker which belongs to the world's largest models of vcibro ripper and almost can broke level 8 hardness rock has entered the stage of the whole machine performance test. Its production can achieve 3 to 5 times of any kind of same level excavator that install the piston breaker and only $70 \%$ of the overall fuel consumption with the suitable working condition. In order to meet the rising demand and expand market, it needs to optimize and improve the structure of SV-80 as soon as possible and launch it in the market at full speed of research and manufacture. It will be a great significant to improve the efficiency and security of construction in crushing industry.

\section{References}

[1] Cao Guojun. A new vcibro ripper:China, 201310178580. 9[P]. 2011-4-1.

[2] Si Kuimou. Development Situation and Research of Hydraylic Breaking Hammer[J]. Equipment Management \&Maintenance Technology. 2009(12):76-77.

[3] Yang Guoping. Research on the new-type hydraulic breaking hammer $[\mathrm{J}]$. Construction Machinery, 2009 (10): 104-106.

[4] Shi Xiuyun. Rotary drilling rig with multiple work method YTR80[J], 2009 (10): 104-106.

[5] Li Yshan. Failure Analysis and Maintenance Measures of Hydraulic Breaking Hammer[J]. Meishan Science, 2013(6): 42-44.

[6] Yang Guoping. Improved design and 3D modeling of YC70 hydraulic impactor[J]. Machinery Design $\varepsilon$ Manufacture, 2011 (5): 41-42.

[7] Trouble shooting of pumping system for concrete pump truck[J]. Mechanical Maintenance, 2004 (11): :102-103.

[8] Cao Guojun. Double motors in vcibro ripper:China, 201310740918. 5 [P]. 2013-12-29.

[9] Peng Zuhuai. Vcibro ripper cutting board:China, 201120318286. X[P]. 2011-8-29.

[10] Xu Jian. Development and Application of Hydraulic Hammer[J]. Reviews and Monographs, 2009 (12): 32-35.

[11] Chen Guoyong. Installation and Maintenance of Broken Hammer[J]. Shenhua Science, 2013, 11 (2): 11-13.

[12] Xu Tongle. Study of Technology Questions in Hydraulic Hammer[J]. Coal Mine Machinery, 2008, 29 (12): 8-10. 